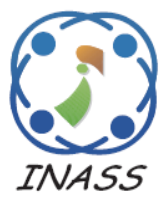

\title{
Enhancement of the Performance of DWDM Free Space Optics (FSO) Communications Systems Under Different Weather Conditions
}

\author{
Salah Mahdi Hamzah ${ }^{1 *}$ \\ Ibrahim A. Murdas ${ }^{1}$ \\ ${ }^{I}$ Department of Electrical Engineering, \\ University of Babylon, College of Engineering, Iraq \\ * Corresponding author's Email: salahmahdee86@gmail.com
}

\begin{abstract}
Frees Space Optical (FSO) communication is one of the communication systems types that uses a free space to transmit information carried by light. It is previously known that the single input single output (SISO) technique is susceptible to atmospheric attenuation because of the effect of weather conditions. The proposed system is a combination of dense wavelength division multiplexing (DWDM) and multiple input multiple output (MIMO) techniques with Fork components. The main Fork component is configured in the Optisystem simulator to connect to another Fork components to increase the power of transmitted signals and ensure the arrival of the transmitted signals to the receiver of the FSO system. The key idea in the proposed system is to mitigate the attenuation that will be happened to the transmitted optical signal which propagated through free space due to different weather conditions. The study is done based on using the Optisystem simulation toolbox that is used to emulate different weather attenuation conditions in two types of FSO systems. The first is the traditional DWDMSISO and the second is a proposed system named Hybrid DWDM-MIMO. A comparison between the proposed system and the traditional system is made in terms of the quality factor under different weather attenuation. The proposed system shows promising results in the performance and the quality of the received signal. The transmission path length of the proposed system under dense fog attenuation of $260 \mathrm{~dB} / \mathrm{km}$ is enhanced to (30.43\%) in comparison with traditional DWDM-SISO, also transmission path length of the proposed system under heavy rain attenuation of $9.29 \mathrm{~dB} / \mathrm{km}$ is enhanced to (55.55\%) in comparison with traditional DWDMSISO. The transmission path length of the proposed system under heavy dry snow of attenuation of 131.835 $\mathrm{dB} / \mathrm{km}$ is enhanced to $(26.19 \%)$ in comparison with traditional DWDM-SISO.
\end{abstract}

Keywords: Free space optics (FSO), Multiple input multiple output (MIMO), Wavelength division multiplexing (WDM).

\section{Introduction}

FSO communication systems have arisen as a technical revolution in wireless communication. Recently, this type of communication system is considered a perfect mean for high rate signal transmission because it depends on the light propagation in transmission. FSO systems have many features over radio frequency (RF) communication systems because of FSO's high bandwidth that is an essential feature in mobile communication. Many drawbacks the RF communication system has, such as its need for time to get the license of spectral frequency. Also, RF system is less secure in comparison with FSO communication system. FSO frequencies operate at near-infrared (IR) therefore indicates to the line of sight technology. FSO communication system can produce potential solutions to the last-mile problem in different ways as FSO has the benefit of its high data rate and flexibility. The FSO communication system is a way by which one sends a modified beam of infrared or visible light across the atmosphere of broadband applications [1]. FSO has the possibility of enhancements and optimization in channel usability field and economical fields in wireless communications $[2,3]$. 
The concept of FSO was studied a long time ago but has not been implemented in practice because the constraints of electronic devices that were developed in that era [4]. FSO communication system has been challenged by attenuation due to object obstacles and weather conditions. Conditions of Weather, such as fog, rain, and snow are the main reasons which impact the transmitted optical signals and cause absorption and scattering. Moreover, object obstacles such as birds or insects cause an interruption to the transmitted optical signal $[5,6]$. Accordingly, recent literature addressed the attenuation and obstacles problems.

A research study proposes using multiple transmission beams between transmitter and receiver stations, i.e., multiple-input multiple-output (MIMO) technique to mitigate the attenuation [7]. In [8], a laser beam combination technology is proposed for multiple beam FSO system, the technology mitigates the problems that emerged from weather attenuations (absorbing, scattering) and losses that are happened in the received power due to losses that occur in the detector. In [9], the study focuses on using MIMO technology to maintain the perfect availability of transmission paths and mitigate weather conditions in the coastal area where the visibility is low due to coastal fog. In [10] the authors made a hybridization configuration of optical amplifiers between erbium doped fiber amplifier (EDFA) and semiconductor optical amplifier (SOA) to mitigate the attenuation of rain. In [11], the authors considered changing the diameter of apertures for transmitters and receivers to get the best performance of the system. In [12], the study proposes amplifying the optical signal by using an optical amplifier to enhance the performance of the transmission system. In [13], the study focuses on enhancing the FSO communication system under sand storms using dual FSO communication channels with EDFA optical amplifier to each one.

The limitations of the aforementioned literature that they did not take into consideration the growing demand in the capacity and scalability of the communication system. Moreover, the previous studies didn't take the impacts of all conditions of weather on the overall system performance.

This study proposes an FSO hybrid DWDM MIMO system that can overcome the problems of atmospheric turbulence with scalability and high data rate. Also, the study compares between the traditional DWDM-SISO system and the proposed system Hybrid DWDM-MIMO and how the proposed system shows a great enhancement in the quality factor of the received signal, transmission link between transmitter and receiver, power of the optical signal and increase scalability. The proposed system is the best candidate to treat a fast increase in capacity without the need for new FSO transmitters and receivers and solve the problem of the last mile.

This work aims to produce a prototype transmission system to transmit optical signals wirelessly under various weather conditions fog, rain and snow and exceed obstacles. The key idea and contributions of this paper are outlined as follow:

1. The proposed system increases the transmission path length of the optical signal with a highquality factor and minimum bit error rate (BER) under normal and severe weather conditions (which cause attenuation to the optical signal).

2. The proposed system which is based on DWDM and MIMO techniques ensures the availability of the transmitted optical signals on the receiver side of the FSO system.

3. The proposed system achieves the requirement of scalability of the number of signals to be transmitted using the DWDM technique.

4. The study shows a detailed comparison between the proposed Hybrid DWDM-MIMO system and the traditional DWDM-SISO, and previous research studies.

The organization of this paper consists of the following: section 2 describes the SISO and WDM techniques and weather conditions such as fog, rain, and snow that cause attenuation. Section 3 describes the proposed system Hybrid DWDM-MIMO. In section 4 discussion and results are presented. Finally, section 5 represents the conclusion.

\section{Background fundamental}

\subsection{SISO technology}

The emerged of wireless communications imparted different technological enhancements in the area of data exchange and information transmission. The traditional pattern of wireless communications uses SISO technique. The concept of SISO technique where only one antenna is used for the transmitter and only one antenna is utilized at the receiver as shown in Fig. 1. FSO system that is used SISO technique is more susceptible to atmospheric attenuation because the effect of weather conditions and interruption due to obstacles like birds and insects.

\subsection{Wavelength division multiplexing (WDM)}

WDM is utilized to enhance the performance of the FSO communication system by increasing the scalability and capacity of the communication system. WDM gets its fame in the field of optical fiber's 


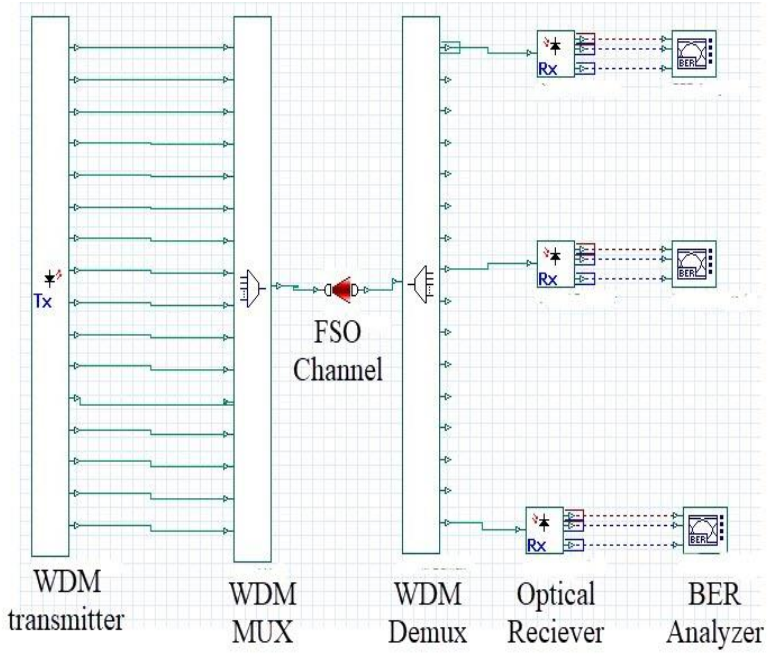

Figure. 1 DWDM-SISO system

communication systems. Two types are known about WDM, (CWDM) coarse wavelength division multiplexing and (DWDM) dense wavelength division multiplexing. The two types are classified according to channel spacing. Also, WDM is a multiplex technology in which more than one optical signal is sent through a single medium using various wavelengths. WDM is a copy of frequency division multiplexing (FDM) but the main variance between the two methods is wavelengths or range of frequencies are utilized in each method [4]. With WDM, multiple data streams are transferred by various optical carriers that have distinguished wavelengths modified and propagated via an optical medium. WDM is utilized to share a great portion of the data optimization usability of the FSO channel. In the WDM system, multiplexers merge a stream of data that are coming from many sources of optical and then project them through optical channel medium.

De-multiplexers, in the last edge of the WDM communication system, recover the data using the technique of de-multiplexing. WDM communication systems allow independent information bitrates and access protocols to work on one system [14] which is a major condition for enhancement of a communication system including FSO systems. This eliminates the cost of combining with changing between protocols that are used because each optical channel that is using a determined protocol can be treated (multiplexed / DE multiplexed) in the ends of the independent transmission system. Therefore, different formats of digital optical information using different bitrates can be sent in their original formats through the same optical channel.

\subsection{Effect of weather conditions on the optical wireless communication}

The greatest challenge to implement optical wireless communication is the weather attenuation which is happened because of scattering and absorption. Water pieces and Carbone dioxide are the main causes of the absorption of wireless optical signals. On the other hand, rain, fog, cloud, and snow are the main reasons that make scattering for wireless optical signals. This scattering makes part of the transmission beam that is transmitted from an optical source to turn around from the desired receiver. The general expression of total attenuation due to weather conditions is represented in Eq. (1).

$$
A_{\text {atm }}=\exp (-\propto L)
$$

Where,

Aatm, denote to total attenuation

$\propto$, denote to the total attenuation coefficient

$L$, denote to transmission path length

The total attenuation coefficient is computed according to Eq. (2).

$$
\alpha=\beta \mathrm{a}+\beta \mathrm{s}
$$

Where,

$\propto$, denote to the total attenuation coefficient $\beta a$, denote to absorption attenuation coefficient $\beta s$, denote to scattering attenuation coefficient The absorption happens due to the variance in the levels of energy and then the heat is emitted. In FSO communication the wavelengths are used to make the change in energy states impossible and the absorption is considered zero [15]. The total attenuation coefficient becomes as in Eq. (3).

$$
\propto=\beta s
$$

$\propto$, denote to the total attenuation coefficient $\beta s$, denote to scattering attenuation coefficient

Based on the particle size of atmospheric weather, the scattering is classified into three major types Mie, Rayleigh, and geometrical scattering [16]. Mie scattering considers the main contributor in FSO attenuation.

\subsubsection{Fog attenuation}

Fog is a cloud of small pieces of water, smoke, ice, or integration between them it's founded near the earth's surface. Fog is the main reason that makes scattering of light to happen which reduces the vision [17]. The limitation of the traditional way to compute 
the fog attenuation is depended on the radius of the particle size. In most cases the size of particle unknown hence it makes it hard to compute the attenuation [5] as in Eq. (4).

$$
\beta_{s}=\sum_{i}^{n \max }\left(n_{i} * Q_{i} \pi r_{i}^{2}\right)
$$

$\beta s$, denotes to scattering attenuation

$n i$, denotes to particle distribution

$Q_{i}$, denotes to scattering efficiency

$r$, denotes to the radius of particle size

To overcome the limitation in the traditional approach, Kim and Kruse introduced a law to find the amount of attenuation depends only on the range of visibility, not on particle size. Atmospheric visibility can be defined is the link distance to the body when the image disparity to $(5 \%)$ from the original image if it is near [18]. The range of visibility is calculated at $550 \mathrm{~nm}$ wavelength that matches the highest intensity of the solar spectrum. The attenuation for both Kim and Kruse model by depending on the range of visibility has been calculated according to Eq. (5) $[5,19]$.

$$
\begin{aligned}
A \text { FOG } & =\frac{10 \log (v \%)}{v k m}\left(\frac{\lambda}{\lambda^{\circ}}\right)^{-q} \\
& =\frac{13}{v}\left(\frac{\lambda}{550}\right)^{-q}
\end{aligned}
$$

Where,

A FOG, denote to fog attenuation in $(\mathrm{dB} / \mathrm{km})$

$v$, denotes to the range of visibility in $\mathrm{km}$

$q$, denotes to particle size distribution coefficient which based on visibility range

$\lambda$, denotes to the wavelength of the transmitted signal in $\mathrm{nm}$

$\lambda^{\circ}=550 \mathrm{~nm}$ denotes to the wavelength visibility reference corresponding to the maximum intensity of the solar spectrum.

$q$, is calculated according to Kim model in Eq. (6).

$$
q=\left\{\begin{array}{lr}
1.6 & \text { if } v>50 \mathrm{~km} \\
1.3 & \text { if } 6 \mathrm{~km}<v<50 \mathrm{~km} \\
0.16 v+0.34 & \text { if } 1 \mathrm{~km}<v<6 \mathrm{~km} \\
v-0.5 & \text { if } 0.5 \mathrm{~km}<v<1 \mathrm{~km} \\
0 & \text { if } v<0.5 \mathrm{~km}
\end{array}\right\}
$$

$q$, is calculated according to Kruse model in Eq. (7).

$$
q=\left\{\begin{array}{lr}
1.6 & \text { if } v>50 \mathrm{~km} \\
1.3 & \text { if } 6 \mathrm{~km}<v<50 \mathrm{~km} \\
0.585 \mathrm{~V}^{1 / 3} & \text { if } v<6 \mathrm{~km}
\end{array}\right\}
$$

Based on Eq. (6) and Eq. (7), the major variation between the two models is the way of how the attenuation is calculated. More specifically, when the visibility is less than $0.5 \mathrm{~km}$, Kruse model unlike Kim model considers the wavelength in calculating the attenuation of the signals in dense fog conditions.

\subsubsection{Rain attenuation}

Rain scattering is formed due to the rainfall when the radius of raindrops ranges between $(100-1000$ $\mu \mathrm{m})$ that is significantly greater than the wavelength of standard FSO systems. As a result, the laser can exceed during the particles of the raindrops with the minimum effect of scattering [20]. The model that is used to calculate attenuation of the rain depends on the rate of rain. The value of the attenuation of the optical signal due to rain also depends on the number of rain droplets and size. The optical signal attenuation linearly raises when the rate of rain increases. However, the average of the rainfall rate increases when the raindrop sizes increases [21]. The attenuation of the wireless optical link which caused by rain is given in Eq. (8) [22].

$$
\text { Arain }=1.07 R^{\frac{2}{3}}
$$

Where,

A rain, denotes to attenuation of the rain in $(\mathrm{dB} / \mathrm{km})$ $R$, denotes to rain Rate in $(\mathrm{mm} / \mathrm{h})$

\subsubsection{Snow attenuation}

Snow pieces are mostly greater than drops of rain and make attenuation to the optical signal greater than effect by the rain. In the literature, the diameter of snow pieces is reported to be more than $20 \mathrm{~mm}$ which is found to be the main reason for a path link to fail when the laser beam is narrow [19]. The energy of the received signal depends on the diameter of snow pieces, the transmission link from the transmitter, and the position of the snow pieces relative to the crosssection of the laser beam [23]. The attenuation of signal caused by snow weather conditions is a function snowfall rate in $(\mathrm{mm} / \mathrm{h})$ and signal wavelength [24]. The attenuation due to snowfall is modelled bases on the classification in wet and dry snow kinds, the attenuation due to snow is calculated in Eq. (9) [24].

$$
\text { A snow }=a S^{b}
$$

Where,

A snow, denotes to attenuation of snow in $(\mathrm{dB} / \mathrm{km})$. 


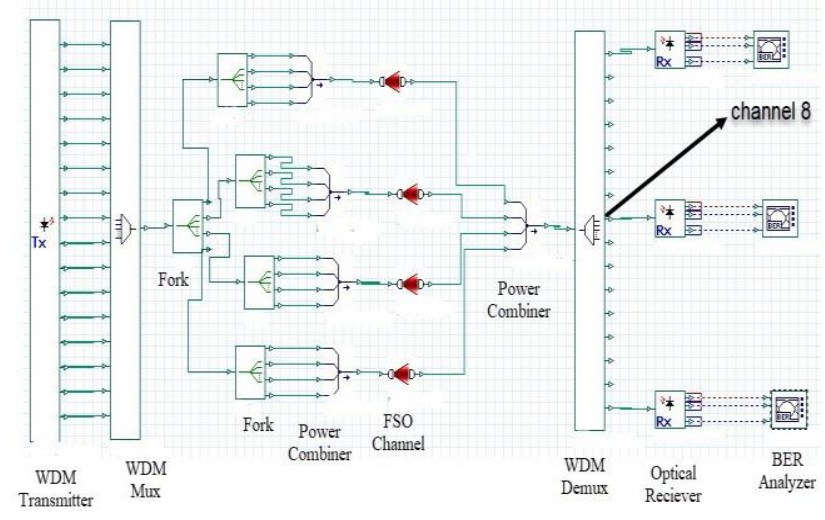

Figure. 2 Hybrid DWDM-MIMO system

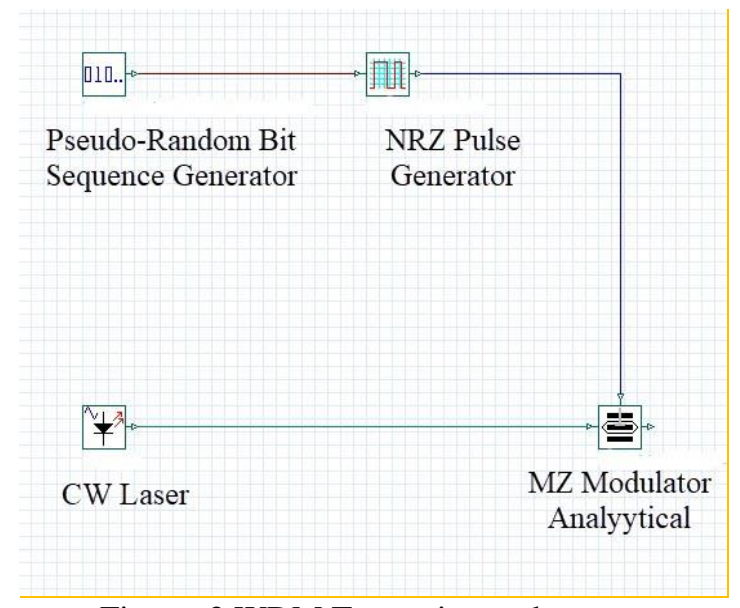

Figure. 3 WDM Transmitter subsystem

$S$, denotes to snowfall rate in $(\mathrm{mm} / \mathrm{h})$

$(a, b)$, denotes to parameters that classify the type of snowfall either wet or dry snow and according to the two types the following values for $(a, b)$ parameters for these types are:-

- For wet snow: $a=1.023\left(10^{-4} \lambda\right)+$ $3.7855466, b=0.72$

- For dry snow: $a=5.42\left(10^{-5} \lambda\right)+5.4958776$, $b=1.38$.

From above the attenuation due to dry snow is greater than wet snow, so it's considered in this study.

\section{Proposed system}

Fig. 2 illustrates the schematic diagram of the proposed system, Hybrid DWDM-MIMO where many antennas at transmitter and receiver are used. The schematic diagram explains three major parts: the transmitter, channel, and receiver. The transmitter implemented by WDM transmitter and WDM multiplexer (WDM Mux). The WDM transmitter consists of 16 subsystems each subsystem includes the following components as shown in Fig. 3:-

1- (CW Laser) Power source: The power source is represented by lasers diode that has a narrow beam width which provides a continuous wavelength of the laser signal. The laser signal is utilized as a carrier to modulate the input electrical signal.

2- Pseudo-Random-Bit-sequence (PRBS) generator: This part responsible of generate random binary sequence. The PRBS generator produces binary sequence from zeros and ones.

3- Non-return to zero (NRZ) generator: This component is a pulse generator that is used for encoding the bit sequence that has been generated by PRBS generator.

4- Mach-Zehnder modulator (intensity modulator): This modulator is utilized to modulate the electrical signal by using a light signal.

WDM multiplexer (WDM Mux) is the second part of the transmitter that is used to multiplex different optical signals wavelengths over one optical communication channel. As shown in Fig. 2, at the end of the WDM multiplexer, a component called Fork is used. Fork component is used to provide multiple beams of the laser from one source of the laser. Every beam of multiple lasers that have been produced by fork is in the same value with the input laser source from the previous component. A group of forks is added to the end of the first fork before transmitting the optical signal in FSO channel to produce multiple laser beams. To enhance the power of the optical signal, beams of lasers produced by these forks are combined using a power combiner and then send in the FSO channel.

The used power in the transmitter is $20 \mathrm{dBm}$. While the frequency of the optical source is 193.1THZ and the spacing between optical channels is $100 \mathrm{GHZ}$. The aperture of the transmitter is $5 \mathrm{~cm}$ and the receiver aperture is $20 \mathrm{~cm}$, and the bit rate is 500 Mbps.

The FSO channel comprises of $4 \times 4$ MIMO technique having various attenuations for different weather conditions.

The receiver part consists of the following components as shown in Fig. 2:-

1- Power combiner is utilized to integrate transmitted optical signals and then injected in WDM DE multiplexer then reverse of transmission operation begin.

2- WDM DE multiplexer (WDM Demux) is used to DE multiplex the received optical signals. In other words, it reverses the function of the WDM multiplexer. 


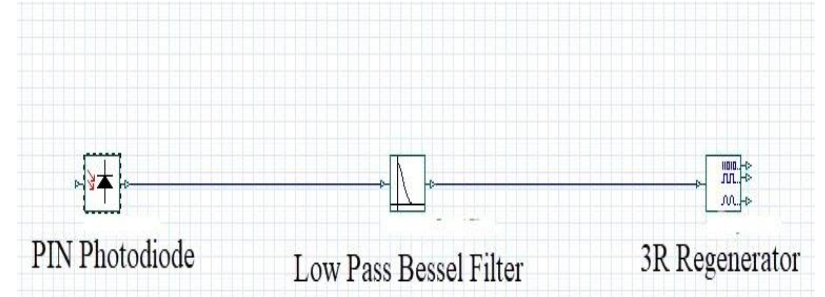

Figure. 4 Optical receiver

3- Optical receiver: After the operation of DE multiplexer, the photo signal enters the optical receiver. The optical receiver as shown in Fig. 4 which consists of PIN photodetector that is used to detect and convert the photo signal into an electrical signal. The detected electrical signal is applied to a low pass Bessel filter. The low pass Bessel filter which is the second part of the optical receiver is used to split the modulated information from the high carrier frequency. The used low pass Bessel filer also minimizes the noise that is produced in the detection process. 3R regenerator is the third part of the optical receiver that is used to produce original sequence of bit and modulated electrical signal to be applied to BER analysis.

4- The BER analyzer is utilized to compute the BER of the received signal and quality factor and to show the eye diagram.

The proposed system is shown in Fig. 2 is used to show the impact of the fog, rain, and snow on the execution of the optical communication system. The schematic model is validated with the benefits of Optisystem simulator. The proposed system is also used to compare with the traditional DWDM-SISO considering the turbulent atmosphere.

The quality factor of the received signal is calculated under different weather conditions by changing the transmission distance between the transmitted and received stations. The quality factor is related to BER as in Eq. (10).

$$
B E R=\frac{\exp (-Q 2 / 2)}{Q \sqrt{2 \pi}}
$$

Where,

$B E R$, denotes to the bit error rate of the received signal

$Q$, the quality factor of the received signal

\section{Results and discussion}

In this section, results and discussion are presented in detail. We used Optisystem simulator to calculate the quality factor of the received optical signal under three different weather conditions: fog, rain, and snow. According to [25], the central DWDM at the receiver has the maximum amount of nonlinearity and poor performance. Therefore, in this study, channel number 8 shown in Fig. 2 is used to calculate the quality factor. In this study, the quality factor of 6 is considered which corresponds to acceptable minimum BER of $10^{-9}$ according to Eq. 10. It is worth noting the study also considers the quality factor values that are below 6 and BER values that are above $10^{-9}$ indicate an ineffective optical communication system. The quality factor of the received signal under different weather conditions is presented in the following sections using both the traditional DWDMSISO and the proposed Hybrid DWDM-MIMO system.

\subsection{Performance of the traditional system DWDM-SISO and the proposed system Hybrid DWDM-MIMO under clear sky and fog weather conditions}

This section examines the effect of clear sky and fog weather conditions on the performance of the DWDM-SISO system and proposed system Hybrid DWDM-MIMO as shown in Fig. 5.

We utilized the mathematical model of Kim and Kruse model Eq. (5) to calculate the attenuation based on different visibility range of weather conditions. For clear sky, the range of visibility is more than $10 \mathrm{~km}$ which corresponds to an attenuation of $0.338 \mathrm{~dB} / \mathrm{km}$. For light fog, the range of visibility is $(0.5<v<10) \mathrm{km}$ which corresponds to an attenuation of $7.743 \mathrm{~dB} / \mathrm{km}$. For moderate fog, the range of visibility is $(0.05<v<0.5) \mathrm{km}$ which corresponds to an attenuation of $26 \mathrm{~dB} / \mathrm{km}$. For dense fog, the range of visibility is $(\mathrm{v}<0.05) \mathrm{km}$ which corresponds to an attenuation of $260 \mathrm{~dB} / \mathrm{km}$.

Given the calculated attenuation values for clear sky and different Fog weather conditions, the Optisystem simulator is used to calculate the quality factor based on different transmission path lengths. The results have been obtained from this simulation are illustrated as follow:

\section{- Clear sky attenuation of $0.338 \mathrm{~dB} / \mathrm{km}$}

As shown in Fig. 5 a, in the traditional DWDM-SISO system, the minimum quality factor (6) is achieved at $2250 \mathrm{~m}$, while in the proposed system, the minimum quality factor is achieved at $7450 \mathrm{~m}$ as shown in Fig. 5 b.

- Light fog attenuation of $7.743 \mathrm{~dB} / \mathrm{km}$ 
As shown in Fig. 5 a, in the traditional DWDM-SISO system, the minimum quality factor (6) is achieved at $1510 \mathrm{~m}$, while in the proposed system, the minimum quality factor is achieved at $2430 \mathrm{~m}$ as shown in Fig. 5 b.

- Moderate fog attenuation of $26 \mathrm{~dB} / \mathrm{km}$

As shown in Fig. 5 a, in the traditional DWDM-SISO system, the minimum quality factor (6) is achieved at $600 \mathrm{~m}$, while in the proposed system, the minimum quality factor is achieved at $1055 \mathrm{~m}$ as shown in Fig. $5 \mathrm{~b}$.

\section{- Dense fog attenuation of $260 \mathrm{~dB} / \mathrm{km}$}

As shown in Fig. 5 a, in the traditional DWDM-SISO system, the minimum quality factor (6) is achieved at $115 \mathrm{~m}$, while in the proposed system, the minimum quality factor is achieved at $150 \mathrm{~m}$ as shown in Fig. 5 b. This means the proposed system increases $30.43 \%$ of the transmission path length of the traditional DWDM-SISO system.

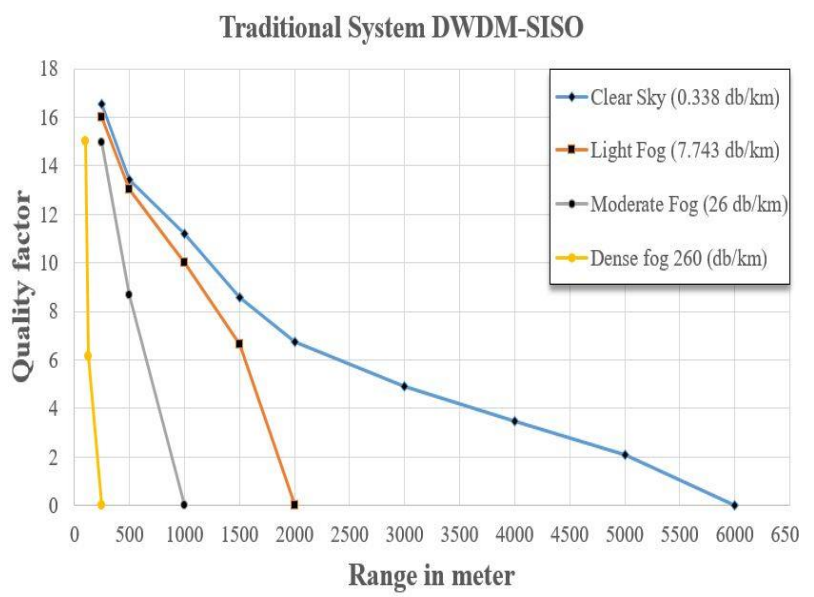

(a)

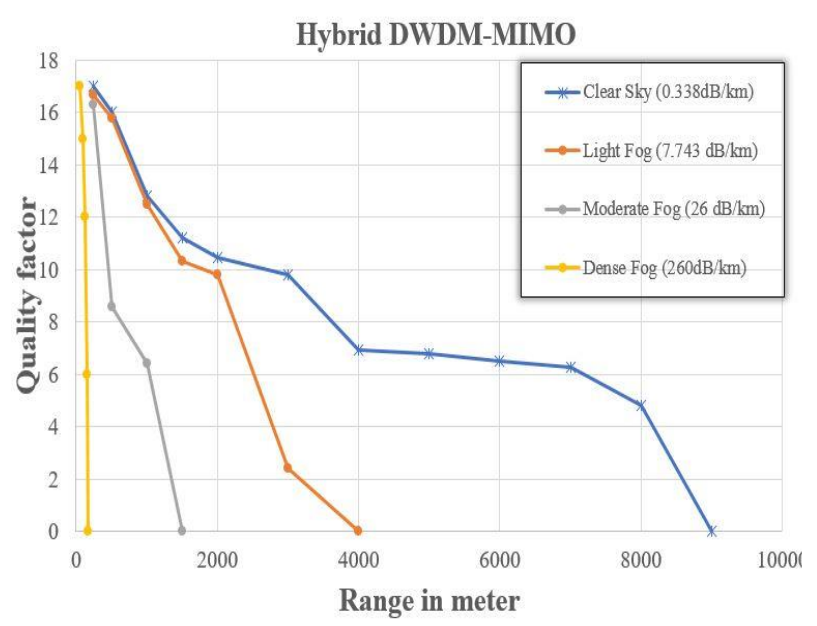

(b)

Figure. 5 Quality factor vs transmission path length using: (a) the traditional DWDM-SISO system and (b) the Hybrid DWDM-MIMO system under a clear sky and fog weather conditions
4.2 Performance of the traditional system DWDM-SISO and the proposed system Hybrid DWDM-MIMO under rainy weather conditions

This section shows the performance of the traditional DWMD-SISO system and proposed system Hybrid DWDM-MIMO under three cases of rainy weather conditions (light, medium, and heavy rain). The attenuation in these cases can be measured according to the Eq. (8) that depend on the rain rate and wavelength of the transmitted optical signal. The average rate of rain for these cases is $2.5 \mathrm{~mm} / \mathrm{h}, 12.5$ $\mathrm{mm} / \mathrm{h}$, and $25 \mathrm{~mm} / \mathrm{h}$, in light rain, medium rain, and heavy rain, respectively [25]. Hence the attenuations for these cases are $1.988 \mathrm{~dB} / \mathrm{km}$ in light rain, $5.8444 \mathrm{~dB} / \mathrm{km}$ in medium rain, and $9.29 \mathrm{~dB} /$ $\mathrm{km}$ in heavy rain.

Given the calculated attenuation values for different Rain weather conditions, the Optisystem simulator is used to calculate the quality factor based on different transmission path lengths. The results have been obtained from this simulation are illustrated as follow:

- Light rain attenuation of $1.988 \mathrm{~dB} / \mathrm{km}$

As shown in Fig. 6 a, in the traditional DWDMSISO system, the minimum quality factor (6) is achieved at $2150 \mathrm{~m}$, while in the proposed system, the minimum quality factor is achieved at 5140 $\mathrm{km}$ as shown in Fig. 6 b.

- Medium rain attenuation of $5.8444 \mathrm{~dB} / \mathrm{km}$ As shown in Fig. 6 a, in the traditional DWDMSISO system, the minimum quality factor (6) is achieved at $1625 \mathrm{~m}$, while in the proposed system, the minimum quality factor is achieved at 2900 $\mathrm{m}$ as shown in Fig. 6 b.

- Heavy rain attenuation of $9.29 \mathrm{~dB} / \mathrm{km}$ As shown in Fig. 6 a, in the traditional DWDMSISO system, the minimum quality factor (6) is achieved at $1350 \mathrm{~m}$, while in the proposed system, the minimum quality factor is achieved at 2100 $\mathrm{m}$ as shown in Fig. 6 b. This means the proposed system increases $55.55 \%$ of the transmission path length of the traditional

DWDM-SISO system.

4.3 Performance of the traditional system DWDM-SISO and the proposed system Hybrid DWDM-MIMO under dry snow weather conditions.

Lastly, this study evaluates the performances of the DWMD-SISO system and proposed system Hybrid DWDM-MIMO in three cases of dry snow 


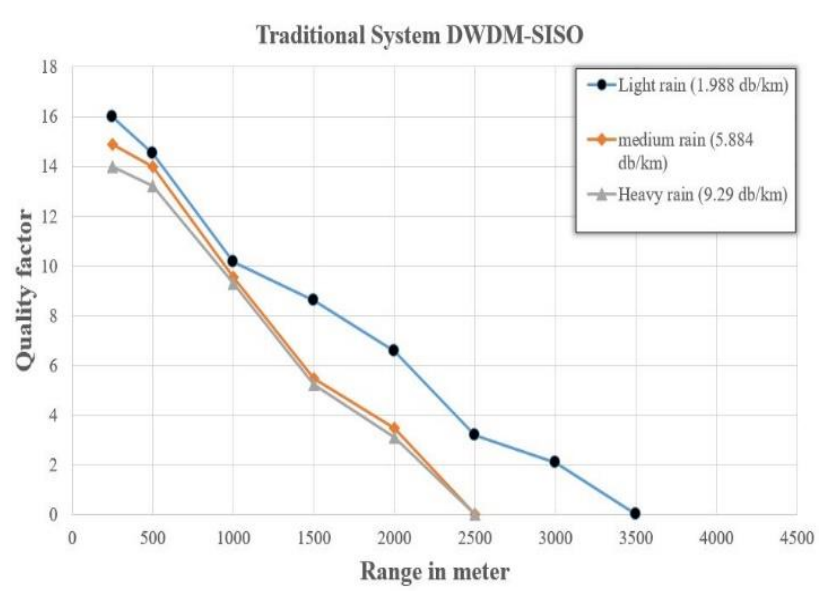

(a)

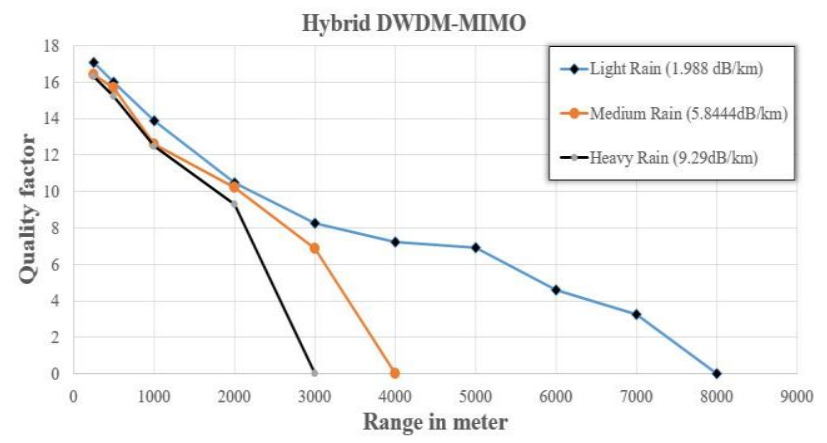

(b)

Figure. 6 Quality factor vs transmission path length using: (a) traditional DWDM-SISO system and (b) hybrid DWDM-MIMO system under rainy weather conditions

weather conditions. The attenuation of the dry snow is calculated based on the Eq. (9) which depends on the snow rate $(S)$. In this study, three types of dry snow conditions are considered which are light, medium, and heavy that are recognized based on the average rate of snow. In light dry snow, the rate of snow is $2.5 \mathrm{~mm} /$ hour and in medium dry snow, the rate of snow is $5 \mathrm{~mm} /$ hour and in heavy dry snow, the rate of snow is $10 \mathrm{~mm} /$ hour [25].

Based on Eq. (9), and using the snow rate, the attenuation values of dry snow conditions are 19.356 $\mathrm{dB} / \mathrm{km}, 50.654 \mathrm{~dB} / \mathrm{km}, 131.835 \mathrm{~dB} / \mathrm{km}$ for light, medium, heavy dry snow respectively.

Given the calculated attenuation values for different dry snow weather conditions, the Optisystem simulator is used to calculate the quality factor based on different transmission path lengths. The results have been obtained from this simulation are illustrated as follow:

\section{- Light dry snow attenuation of $19.356 \mathrm{~dB} / \mathrm{km}$}

As shown in Fig. 7 a, in the traditional DWDMSISO system, the minimum quality factor (6) is achieved at $775 \mathrm{~m}$, while in the proposed system, the minimum quality factor is achieved at 1265 $\mathrm{m}$ as shown in Fig. 7 (b).
- Medium dry snow attenuation of $\mathbf{5 0 . 6 5 4}$ $\mathrm{dB} / \mathbf{k m}$

As shown in Fig. 7 a, in the traditional DWDMSISO system, the minimum quality factor (6) is achieved at $450 \mathrm{~m}$, while in the proposed system, the minimum quality factor is achieved at $600 \mathrm{~m}$ as shown in Fig. 7 b.

- Heavy dry snow attenuation of $131.835 \mathrm{~dB} / \mathrm{km}$ As shown in Fig. 7 a, in the traditional DWDMSISO system, the minimum quality factor (6) is achieved at $225 \mathrm{~m}$, while in the proposed system, the minimum quality factor is achieved at $265 \mathrm{~m}$ as shown in Fig.7 b. This means the proposed system increases $26.19 \% \%$ of the transmission path length of the traditional DWDM-SISO system.

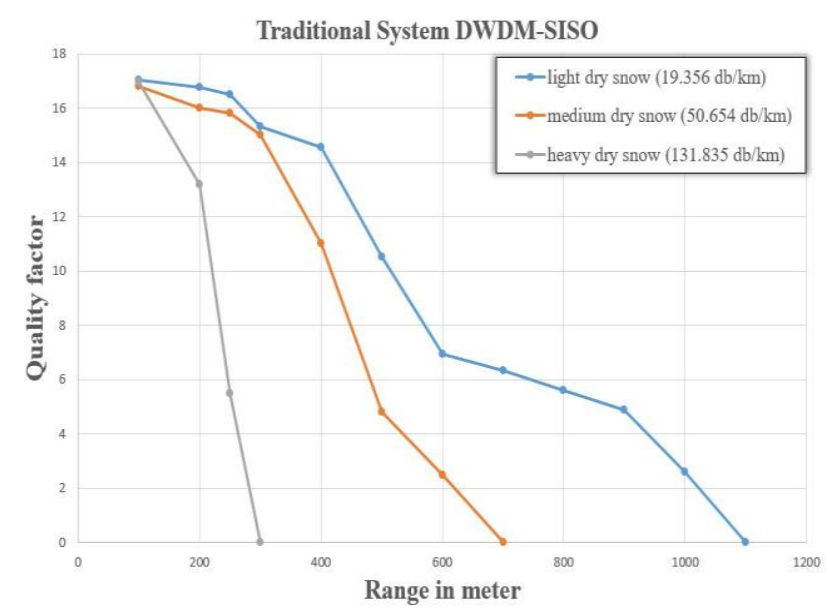

(a)

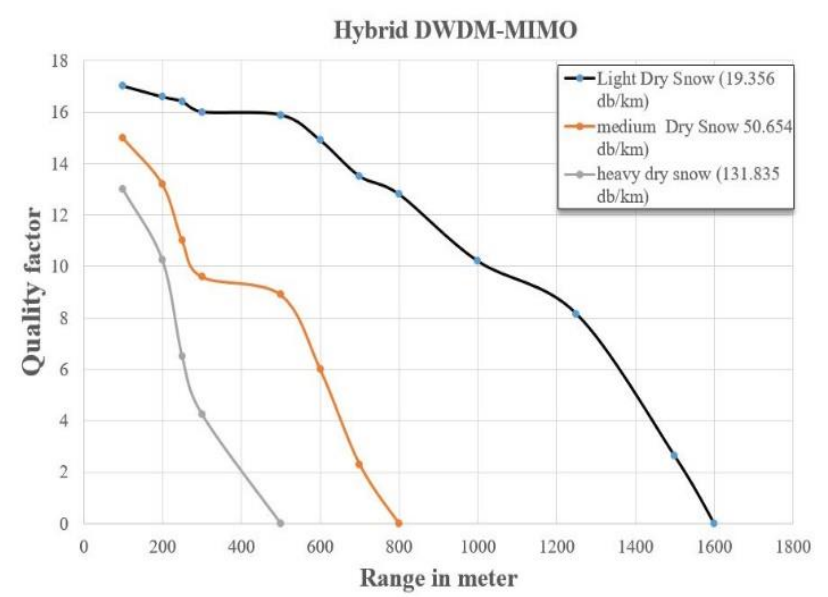

(b)

Figure. 7 Quality factor vs transmission path length using: (a) traditional DWDM-SISO system and (b) hybrid

DWDM-MIMO system under dry snow weather conditions 
Table 1. Comparison between 16-channel traditional DWDM-SISO system and 16-channel Hybrid DWDMMIMO system in maximum transmission lengths in meter that corresponding quality factor (6) and BER $10^{-9}$ under different weather conditions

\begin{tabular}{|c|c|c|}
\hline $\begin{array}{c}\text { Weather } \\
\text { conditions }\end{array}$ & $\begin{array}{c}\text { DWDM- } \\
\text { SISO } \\
\text { system }\end{array}$ & $\begin{array}{c}\text { Hybrid } \\
\text { DWDM- } \\
\text { MIMO system }\end{array}$ \\
\hline Clear sky & 2250 & 7450 \\
\hline Light fog & 1510 & 2430 \\
\hline Moderate fog & 600 & 1055 \\
\hline Dense fog & 115 & 150 \\
\hline Light rain & 2150 & 5140 \\
\hline Medium rain & 1625 & 2900 \\
\hline Heavy rain & 1350 & 2100 \\
\hline Light dry snow & 775 & 1265 \\
\hline Medium dry snow & 450 & 600 \\
\hline Heavy dry snow & 210 & 265 \\
\hline
\end{tabular}

A summary of the achieved results is presented in Table 1. Table 1 summarizes a significant improvement of using the proposed system over the traditional system DWDM-SISO for the optical communication system. The results show the quality factor decreases when the transmission path length increases due to attenuation. However, using the proposed system, the signal can be transmitted for longer path length with a better signal quality under all the described weather conditions. This is achieved due to using the fork component and MIMO technique. Using a group of fork components, the input power is duplicated which led to an increase in the total power of the transmitted optical signal. Increasing the total power of the transmitted optical signal mitigates the attenuation of different weather conditions. The used MIMO technique is produced redundancy transmission paths which help to overcome the problem of obstacles. As a result, the use of the fork component and MIMO technique reduces the attenuation, increases the transmission path length, improves the quality factor of the optical signal, and subsequently, it improves the BER according to Eq. (10).

By comparing the system method with previous studies in the same field, it is possible to note there is a significant improvement in term of transmission path length as shown in Table 2, under same conditions and considering the same lowest acceptable level of quality factor 6 in digital communication which corresponding to BER 10-9. The maximum transmission path length of the proposed system with 16-DWDM, 4× 4 MIMO channel, and channel spacing $100 \mathrm{Ghz}$ achieved a transmission path length of $1350 \mathrm{~m}$ that is greater than $1090 \mathrm{~m}$ the achieved result in [1] under the same
Table 2. Comparison between the proposed work and related previous works

\begin{tabular}{|l|l|l|l|l|}
\hline Author & $\begin{array}{l}\text { Power } \\
\text { in } \mathrm{dBm}\end{array}$ & $\begin{array}{l}\text { Data } \\
\text { rate } \\
\text { In } \\
\text { Gbps }\end{array}$ & $\begin{array}{l}\text { Attenuation } \\
\text { in } \mathrm{dB} / \mathrm{km}\end{array}$ & $\begin{array}{l}\text { path } \\
\text { length } \\
\text { in meter }\end{array}$ \\
\hline $\begin{array}{l}{[1] \text { in }} \\
2014\end{array}$ & 7.7 & 1.25 & 19.2 & 1090 \\
\hline $\begin{array}{l}{[14] \text { in }} \\
2016\end{array}$ & 20 & 2.5 & 2 & 1000 \\
\hline $\begin{array}{l}{[4] \text { in }} \\
2019\end{array}$ & 10 & 2.5 & 4.2872 & 4000 \\
\hline \multirow{2}{*}{$\begin{array}{l}\text { Proposed } \\
\text { work }\end{array}$} & 20 & 2.5 & 2 & 1350 \\
\cline { 2 - 5 } & 10 & 2.5 & 4.2872 & 4700 \\
\cline { 2 - 5 } & & 1.25 & 19.2 & 5000 \\
\hline
\end{tabular}

weather conditions. Also, the maximum transmission path length of the proposed system with 8-DWDM, $4 \times 4$ MIMO channel, and channel spacing $100 \mathrm{Ghz}$ achieved a transmission path length of $5000 \mathrm{~m}$ that is greater than $1000 \mathrm{~m}$ the achieved result in [14] under the same conditions. Further, the maximum transmission path length of the proposed system with 32-DWDM, 4× 4 MIMO channel and channel spacing $100 \mathrm{Ghz}$ achieved a transmission path length of $4700 \mathrm{~m}$ that is greater than $4000 \mathrm{~m}$ the achieved result in [4] under the same conditions. The promising performance of the proposed system is attributed to the combination between the MIMO technique and the DWDM technique. The MIMO technique maintains a perfect availability of transmission path and increases the power of transmitted signals, and the DWDM technique increases the scalability of the number of transmitted signals. It is worth noting that the proposed system with the MIMO technique increases the availability of the transmitted signals and helps in mitigating the impact of interruption due to obstacles like birds, and insects.

\section{Conclusion}

Despite the FSO communication system has main advantages including high data rate, security, and capacity, this technique suffers from challenges mainly because of the various weather effects such as rain, fog, snow, and clouds in the atmosphere of the earth. This paper presents the implementation and simulation of the traditional DWDM-SISO system and the proposed Hybrid DWDM-MIMO system. Each system is tested under different weather conditions, such as the clear sky and 
various fog conditions, various rain conditions, and various dry snow conditions.

The results which are achieved from the proposed DWDM-MIMO show an improvement in the transmission range and signal quality compared to the traditional DWDM-SISO. As indicated in Table 1, the DWDM-MIMO shows a significant increase in the transmission path length and signal quality under different weather conditions and attenuation. Considering the worst weather condition that causes a high attenuation value, the transmission path length of the proposed system under dense fog attenuation of $260 \mathrm{~dB} / \mathrm{km}$ is enhanced to $(30.43 \%)$ in comparison with traditional DWDM-SISO. Also, the transmission path length of the proposed system under heavy rain attenuation of $9.29 \mathrm{~dB} / \mathrm{km}$ is enhanced to $(55.55 \%)$ in comparison with traditional DWDMSISO. Moreover, the transmission path length of the proposed system under heavy dry snow of attenuation of $131.835 \mathrm{~dB} / \mathrm{km}$ is enhanced to (26.19\%) in comparison with traditional DWDMSISO. Further, as indicated in Table 2, the proposed system showed a significant improvement in comparison with other research studies.

It is worth highlighting here the benefit of the proposed system in severe weather conditions. The proposed system can be used in places that have severe weather conditions such as heavy fog, heavy rain, snow, and dust storms. These sever weather conditions cause a high rate of attenuation on the transmitted optical signal. High attenuations affect the characteristics of the FSO communication system and consequently lead to an increase in BER and degradation quality factor to bad levels. The proposed system mitigates these attenuations and ensures the arrival of the transmitted signal to the destination. The study also proves that both the largest transmission path length and quality factor improve when increasing the number of transmitters and receivers of the transmission system. All the over mentioned enhancements are attributed to the proposed combination between the theory of the DWDM and the MIMO technique described in section 3 .

\section{Conflicts of Interest}

The authors declare no conflict of interest.

\section{Author Contributions}

The authorship of this article is based on the following criteria: conceptualization, Salah Mahdee Hamza and Ibrahim A.Murdas; methodology, Salah Mahdee Hamza; software, Salah Mahdee Hamza; validation, Salah Mahdi Hamza, Ibrahim A.Murdas,; formal analysis, Salah Mahdee Hamza; investigation, Salah Mahdee Hamza; resources, Salah Mahdee Hamza; data curation, Salah Mahdee Hamza; writing - original draft preparation, Salah Mahdi Hamza; writing - review and editing, Ibrahim A.Murdas; visualization, Salah Mahdee Hamza; supervision, Ibrahim A.Murdas; project administration, Ibrahim A. Murdas; funding acquisition, N/A", etc.

\section{References}

[1] A. B. Mohammad, "Optimization of FSO System in Tropical Weather Using Multiple Beams", In: Proc. of International Conf. on IEEE Photonics (ICP), pp. 109-112, 2014.

[2] S. Kaur and A. Kakati, "Analysis of Free Space Optics Link Performance Considering the Effect of Different Weather Conditions and Modulation Formats for Terrestrial Communication", Journal of Optical Communication, 2018.

[3] S. Kaur, "Analysis of Inter-Satellite Free-Space Optical Link Performance Considering Different System Parameters", Opto-Electronics Review, Vol. 27, No. 1, pp. 1013, 2019.

[4] A. Ahmed, A. Singh, A. Singh, and S. Kaur, "Performance Analysis of WDM-MIMO Free Space Optical System Under Atmospheric Turbulence", In: Proc. of International Conf. on Signal Processing and Integrated Networks (SPIN), pp. 820-825, 2019.

[5] Z. Mahlobogwane, P. A. Owolawi, and O. Sokoya, "Multiple Wavelength Propagation in Free Space Optical Wireless Channel", In: Proc. of International Conf. on Advances in Big Data Computing and Data Communication Systems (icABCD), pp. 1-6, 2018.

[6] S. A. Al-Gailani, A. B. Mohammad, and R. Q. Shaddad, "Enhancement of Free Space Optical Link in Heavy Rain Attenuation Using Multiple Beam Concept", Optik (Stuttg), Vol. 124, No. 21, pp. 4798-4801, 2013.

[7] N. H. M. Noor, A. W. Naji, and W. Al-Khateeb, "Performance Analysis of a Free Space Optics Link With Multiple Transmitters/Receivers", IIUM Enginnering Journal, Vol. 13, No. 1, 2012.

[8] F. D. Kashani, M. R. Mahzoun, and B. Ghafary, "Beam Propagation Analysis of a Multi Beam FSO System With Partially Flat-topped Laser Beams in Turbulent Atmosphere", Optik (Stuttg), Vol. 123, No. 10, pp. 879-886, 2012. 
[9] N. H. M. Noor, W. Al-Khateeb, and A. W. Naji, "Experimental Evaluation of Multiple Transmitters/Receivers on Free space optics link", In: Proc. of International Conf. on IEEE Student Conference on Research and Development, pp. 128-133, 2011.

[10] G. Sharma and L. Tharani, "Performance Evaluation of WDM-FSO Based Hybrid Optical Amplifier Using Bessel Filter", In: Proc. of International Conf. on Communication and Signal Processing (ICCSP), pp. 653-656, 2018.

[11] V. Sharma and G. Kaur, "High speed, Long Reach OFDM-FSO Transmission Link Incorporating OSSB and OTSB Schemes", Optik (Stuttg), Vol. 124, No. 23, pp. 6111-6114, 2013.

[12] V. Sharma, "High Speed CO-OFDM-FSO Transmission System", Optik (Stuttg), Vol. 125, No. 6, pp. 1761-1763, 2014.

[13] S. H. Alnajjar, A. A. Noori, and A. A. Moosa, "Enhancement of FSO Communications Links Under Complex Environment", Photonic Sensors, Vol. 7, No. 2, pp. 113-122, 2017.

[14] V. Manea, M. Petre, S. Puşcoci, and D. A. Stoichescu, "The Approach of Wavelength Dense Multiplexing Using Free Space Optical Systems", In: Proc. of International Conf. on Electronics, Computers and Artificial Intelligence (ECAI), pp. 1-6, 2016.

[15] S. Bloom, E. Korevaar, J. Schuster, and H. Willebrand, "Understanding the Performance of Free-space Optics", Journal of Optical Networking, Vol. 2, No. 6, pp. 178-200, 2003.

[16] K. Kazaura, "Studies on Performance of Ultra High Speed Free Space Optical Communication Systems", Waseda University, 2007.

[17] M. S. Awan, E. Leitgeb, S. S. Muhammad, F. Nadeem, M. S. Khan, and C. Capsoni, "Distribution Function for Continental and Maritime Fog Environments for Optical Wireless Communication", In: Proc. of International Conf. on Communication Systems, Networks and Digital Signal Processing, pp. 260-264, 2008.

[18] F. Nadeem, V. Kvicera, M. S. Awan, E. Leitgeb, S. S. Muhammad, and G. Kandus, "Weather Effects on Hybrid FSO/RF Communication Link", IEEE Journal on Selected Areas Communication, Vol. 27, No. 9, pp. 1687-1697, 2009.

[19] F. Nadeem, E. Leitgeb, M. S. Awan, and G. Kandus, "Optical Wavelengths Comparison for Different Weather Conditions", In: Proc. of
International Conf. on Satellite and Space Communications, pp. 279-283, 2009.

[20] A. Alkholidi and K. Altowij, "Effect of Clear Atmospheric Turbulence on Quality of Free Space Optical Communications in Western Asia", Optical Communication System, pp. 41, 2012.

[21] M. Achour, "Simulating Atmospheric FreeSpace Optical Propagation: Rainfall Attenuation”, In Free-Space Laser Communication Technologies XIV, Vol. 4635, pp. 192-201. 2002

[22] T. H. Carbonneau and D. R. Wisely, "Opportunities and Challenges for Optical Wireless: The Competitive Advantage of Free Space Telecommunications Links in Today's Crowded Marketplace", In Wireless Technologies and Systems: Millimeter-Wave and Optical, Vol. 3232, pp. 119-128, 1998.

[23] M. Akiba, K. Ogawa, K. Wakamori, K. Kodate, and S. Ito, "Measurement and Simulation of The Effect of Snowfall on Free-space Optical Propagation", Applied Optics, Vol. 47, No. 31, pp. 5736-5743, 2008.

[24] M. S. Awan, Statistical-dynamical Channel Modeling of Outdoor Optical Wireless Links, 2010.

[25] I. A. Murdas and Y. K. Abbas, "Design and Simulation of WDM-UWB Based OWC System”, Jurnal of University of Babylon, Vol. 24, No. 4, pp. 893-906, 2016. 